\title{
The Relationship between 2D Positioning Ability and Realistic Drawing Skill
}

\author{
Shih Ting Huang, I. Ping Chen* \\ Institute of Applied Arts, National Chiao Tung University, Taiwan \\ Email: yap1982@gmail.com, *iping@faculty.nctu.edu.tw
}

How to cite this paper: Huang, S. T., \& Chen, I. P. (2017). The Relationship between 2D Positioning Ability and Realistic Drawing Skill. Art and Design Review, 5, $1-12$.

https://doi.org/10.4236/adr.2017.51001

Received: November 14, 2016

Accepted: December 27, 2016

Published: December 30, 2016

Copyright (c) 2017 by authors and Scientific Research Publishing Inc. This work is licensed under the Creative Commons Attribution International License (CC BY 4.0).

http://creativecommons.org/licenses/by/4.0/ (c) (i) Open Access

\begin{abstract}
Realistic drawing ability is a fundamental human trait. However, the cognitive constituents of this ability are poorly understood. This study aims to evaluate the contribution of Two-dimensional (2D) positioning skills in the performance of realistic drawing. 2D positioning refers to an important observation technique employed by artists to locate the correct position of an object in its representation on a blank canvas. Two different types of $2 \mathrm{D}$ positioning techniques are considered: absolute positioning (using the canvas boundaries as positioning reference) and relative positioning (using already-drawn components as reference). We used a line-arrangement experimental task to evaluate the positioning abilities of experts and novices; we further measured their realistic drawing ability in a realistic drawing task. Pearson correlation-coefficient analysis showed that both positioning abilities are positively correlated with realistic drawing abilities, but that the two positioning abilities are not correlated. While expert participants were found to be significantly better at absolute positioning than the novice, their relative positioning performance does not differ from that of the novice. These results suggest that absolute and relative positioning are two independent observation techniques, and that experience might improve absolute positioning skills. This implies that absolute positioning is a more advanced technique requiring both training and practice.
\end{abstract}

\section{Keywords}

2D Positioning, Absolute Positioning, Relative Positioning, Realistic Drawing Skill

\section{Introduction}

Mastering realistic drawing takes long years of practice. It is a skill in which even experienced artists are still in the process of refining. Furthermore, realistic por- 
traits exhibit obvious differences between artists. It is therefore worth investigating what cognitive abilities are involved in the creation of realistic drawings.

The production of a realistic drawing involves a series of complicated actions, including visual processing and motor skills. The specifics of this process have yet to be elucidated by the scientific community. Cohen and Bennett (1997) identified four phases involved in the completion of a realistic drawing. Phase 1 involves observation of the target object. In Phase 2 the artist determines which part (of the whole) will be represented and what method she/he will employ to represent the object. Phase 3 requires eye-hand coordination to represent what she/he observes. Phase 4 requires objective evaluation of the finished product and effective revision. Their research results suggest that the key to success in realistic drawing lies in astute observation, which is believed to increase the effect of realism in a representation. The quality of observation is negatively affected by the illusions and delusions maintained by the observer. Illusions refer to "perceptual constancies", which affect performance even to the point of inaccuracy (Cohen \& Earls, 2010). The size, shape, color, and brightness of an object might all be affected by perceptual constancies (Cohen \& Jones, 2008; Day, 1972; McManus, Loo, Chamberlain, Riley, \& Brunswick, 2011; Mitchell, Ropar, \& Ackroyd, 2005; Ostrofsky, Kozbelt, \& Cohen, 2015; Ostrofsky, Kozbelt, \& Seidel, 2012; Perdreau \& Cavanagh, 2011; Todorovic, 2002, 2010). Delusions pertain to an artist's knowledge of the object under observation, since this prior knowledge tends to guide an artist in her/his representation. Kozbelt, Seidel, El Bassiouny, Mark and Owen (2010) explored visual attention from the perspective of delusions. Other researchers have investigated the relationship between "frequency of observation" and art creation. Cohen (2005) suggested that frequent observation allows for a high-quality working memory of the observed object, which ensures the accuracy of its representation. Cohen and Bennett (1997) were the first researchers to establish the existence of a correlation between correct observation and realistic representation.

While different approaches such as top-down or bottom-up might produce different observations, it is the observer's ability which plays the most important role in drawing. Carson and Allard (2013) examined whether an artist's ability to correctly observe the angle of an object affects the representation of realistic drawing. Results indicate that the more experienced an artist is, the better she/he is able to depict the angle of the target object. A highly experienced artist can draw all angles with the same accuracy, whereas a less experienced drawer would find some angles harder to depict than others.

This study is focused on another factor of observation as yet unexplored: perception of the location of an object. When working on a realistic artwork, the first thing is to find out the right position and create a truthful composition on canvas. This can be achieved by a multitude of methods. For example, we may use our hands as a viewfinder with which to compose a real-life picture and thus set referential positions for any objects inside the "window". We also need a referential system by which we are able to locate the correct position of any objects 
on the canvas and thus make a truthful representation. This technique is called "2D positioning". There are several approaches to achieve 2D positioning throughout the drawing process. The artist may use the boundary of the canvas as the referential system in selecting an appropriate starting point. This is called "absolute positioning". As more lines and objects are represented on the canvas, there are a larger number of components which can be employed as references to locate succeeding objects. This is called "relative positioning".

The above two techniques are commonly adopted by artists attempting to draw a realistic drawing. We aimed to ascertain the interplay of abilities in absolute positioning, relative positioning, and realistic drawing. In addition, drawing or painting experience also affects retrieval of visual information and the level of accuracy and precision of realistic drawing. This suggests that the difference between a novice and an expert lies not only in drawing skills, but also in their cognitive abilities (e.g., Cohen \& Bennett, 1997; Cohen \& Jones, 2008; Kozbelt, 2001; Kozbelt \& Seeley, 2007; Mitchell, Ropar, Ackroyd, \& Rajendran, 2005; Schlewitt-Haynes, Earthman, \& Burns, 2002). The literature indicates that previous drawing experience might have an impact on $2 \mathrm{D}$ positioning techniques; this study aims to confirm that correlation.

\section{Method}

We designed three tasks to measure three dimensions of art creation: absolute positioning, relative positioning, and realistic drawing. All participants were involved in all three tasks. The first two tasks aimed to assess a drawer's absolute and relative positioning abilities. The scope of measurement in the positioning tasks is observation by eye, and excludes other factors such as skill of motion control by wielding a pen, eye-hand coordination, and shape constancy. In a series of computer-aided tests, participants were required to place lines displayed on the interface in the right locations. In the process, they could only check the locations of these lines by eye while moving the mouse upward and downward to change the lines on the interface. Participants did not use brushes to copy these lines. The "absolute positioning" task was designed with a frame over the interface that resembles the boundary of a canvas, so participants could use the frame as a referential point. In the "relative positioning" task participants moved a group of lines on the interface with reference to each other, as if they were doing jigsaw puzzles. The "realistic drawing" task required representation of still-life objects, and realism was evaluated in an ecologically valid context. The result of this measurement was correlated with abilities in absolute positioning and relative positioning. Details regarding these data acquisition tasks are provided below.

\subsection{Participants}

We selected 51 participants from college and graduate institutions. There were 28 females and 23 males, with an average age of 23.2 years $(S D=2.73)$. We divided them into two groups according to their drawing experience. Among 
them, 19 participants were categorized as "experts", who either have received professional training in art creation, or are students in design fields and whose hobbies include drawing or painting. The other 32 participants were categorized as "novices".

\subsection{Task \#1: Measurement of Absolute Positioning Ability}

This task measures the ability to apply absolute positioning, which refers to the ability to use the boundary of the canvas as a referential point to locate the position of the object to be drawn. A fixed black-line frame was used to indicate the boundary of the canvas. Participants were expected to move the lines inside the frame to their correct locations.

\subsubsection{Materials}

Participants were required to use desktop computers (Windows 7, 19" LCD screen (EIZO S2133-H)) to perform the tests, and a keyboard and a mouse was provided to navigate the interface. Using FLASH as the programming language, we created a two-toned inter-face over a LCD screen. Two identical black-line frames are displayed on a white back-ground (shown in Figure 1), which is 340 pixels $\times 474$ pixels with the visual angle is $13.7^{\star} 19.0$. The left frame serves as the referential zone, while participants operate on the right frame. The two frames are not placed on the same horizontal surface so as to increase the level of difficulty of the task.

To start, a single line would appear in the referential zone. This represents the moment an artist takes up his pencil or brush to make his first mark. In the operating zone, a similar line would be shown at a different location and angle. Participants were required to move the line in the operating zone until it matched
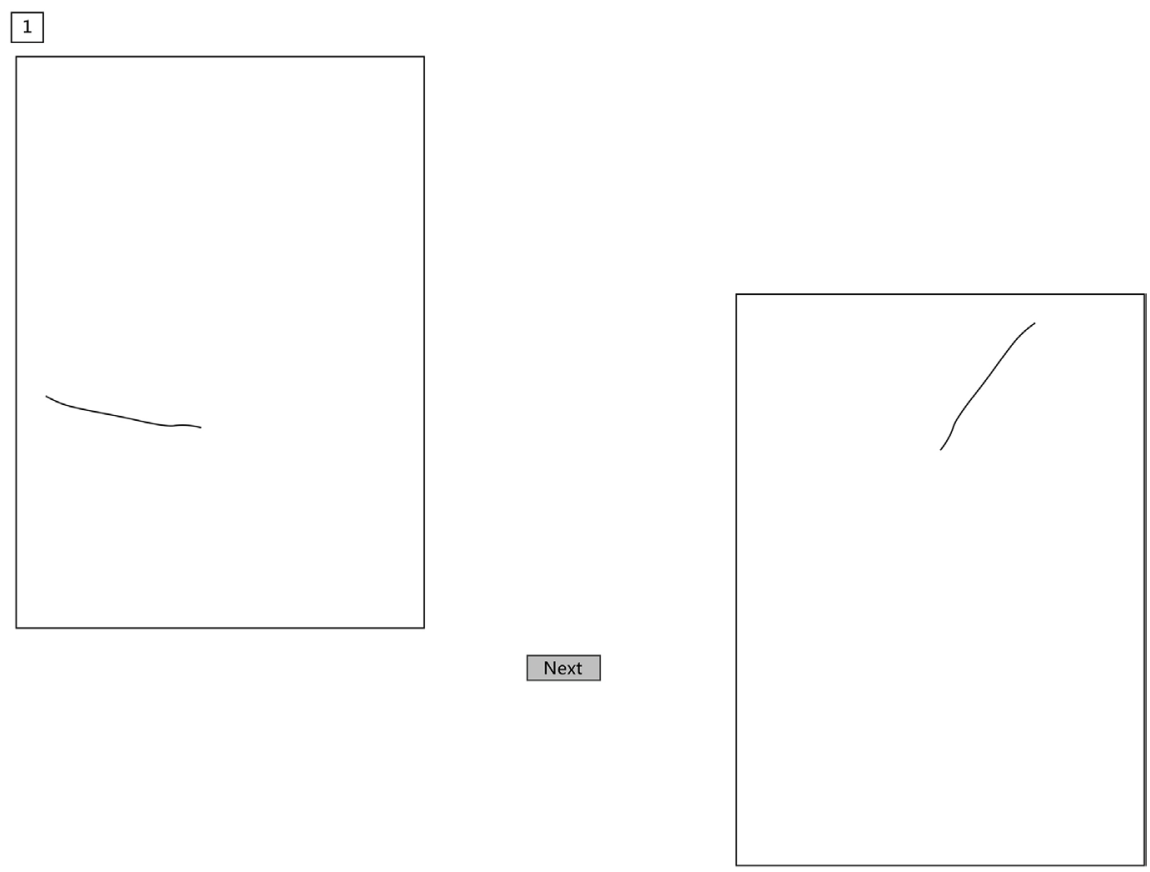

Figure 1. User interface for Task \#1. 
the location and angle of the line in the referential zone. The participant clicks on a mouse with his right hand to select the line, while his left hand presses the arrow keys on the computer keyboard to adjust the angle of the line. Once the line is matched in terms of position, the participant presses the "Next" key and starts to move the next line. A total of 60 lines are presented. They differ in length, orientation, and shape. All black lines are organic curves ranging between 18 and 268 pixels (visual angle between degrees 0.7 - 10.8).

\subsubsection{Procedure}

Participants were required to move the line within the operating zone as quickly as possible until the two lines look identical. This had to be done by eye; hands, rulers and other tools were prohibited. They were not explicitly told that the black-line frame can be used as a referential point for positioning.

\subsection{Task \#2: Relative Positioning Ability}

We hypothesized that artists tend to use already-drawn lines and objects as referential points to anchor their starting point. This task aims to measure the ability to apply relative positioning. In this task the lines comprising an image are scrambled, and participants are required to move them to the right position, similar to a jigsaw puzzle. They can use any lines already in their correct position as clues to determine which line to move next.

\subsubsection{Materials}

The equipment used in this task was the same as that used in Task \#1. This program was also written in the FLASH programming language. We created a two-toned interface on a 19" LCD screen (EIZO S2133-H). Two identical black-line frames are displayed (Figure 2) inside which 20 - 30 random lines are shown on a white background. Participants were asked to put a pattern or figure

1

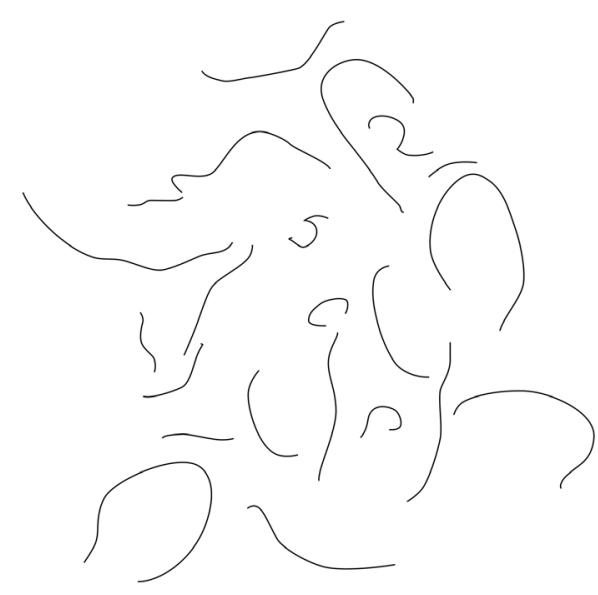

Figure 2. User interface for Task \#2. 
back together (Figure 3): a bird on a branch (comprising 33 lines), a human face (comprising 27 lines) and the head of a cow (comprising 22 lines). The visual angle is $19.3^{\star} 14.8$ for a complete image. We chose images of animals and humans over human-made objects mainly because they are made up of organic curves with fewer straight lines. This poses a greater challenge for participants.

\subsubsection{Procedure}

Participants were given a printed A3 image. They were asked to use a computer mouse to move the irregular lines on the computer screen to recreate the printed image. The print is rendered at $170 \%$ of the computer image (visual angle at $32.8^{\star} 25.2$ degrees) so participants had to make a conscious effort to trace the exact position.

Participants were asked to recreate the original pattern by eye as quickly as possible. The print was placed on the desk, and could not be held nearer to the screen as a reference. Participants could use the mouse and left and right keys on the keyboard to adjust the direction of the lines.

\subsection{Task \#3: Realistic Drawing Ability}

This task was designed to measure the participant's drawing ability in the format of still-life drawing that conforms better to ecological validity than the two previous tasks.

\subsubsection{Materials}

There are two groups of still-life objects. This first is made up of four apples (left inset of Figure 4). The four apples are of different varieties, thus exhibiting differing textures, size, and color. They were randomly arranged at varying depths, which means participants must pay close attention to bring out a sense of space. The second stimulus was a doll $(50 \mathrm{~cm})$. It is of slightly different proportions to those of an average human, and was placed in an exaggerated posture. This stimulus is far more complicated than the apple stimulus. The various components (curly hair, flannel, gauze, and body of the model) pose a much higher degree of challenge.

\subsubsection{Procedure}

Participants were asked to faithfully represent the two still-lifes. They were given an hour to complete each drawing.
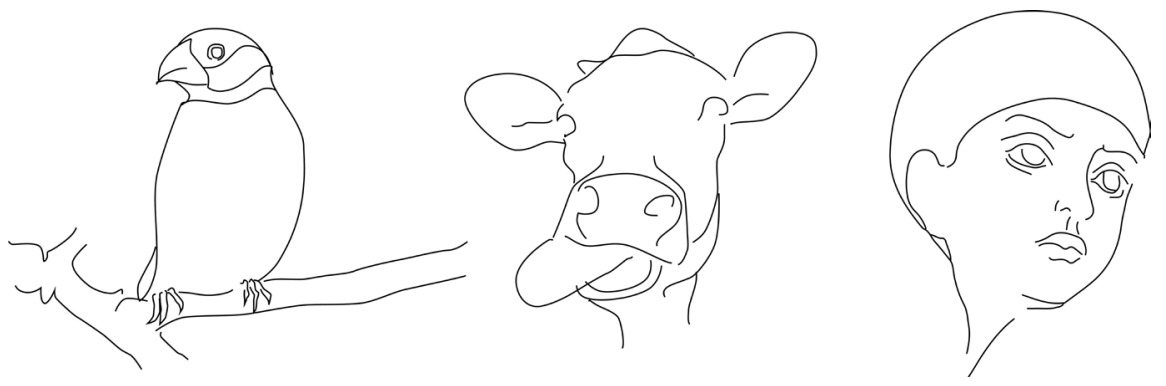

Figure 3. Stimuli used in Task \#2. 


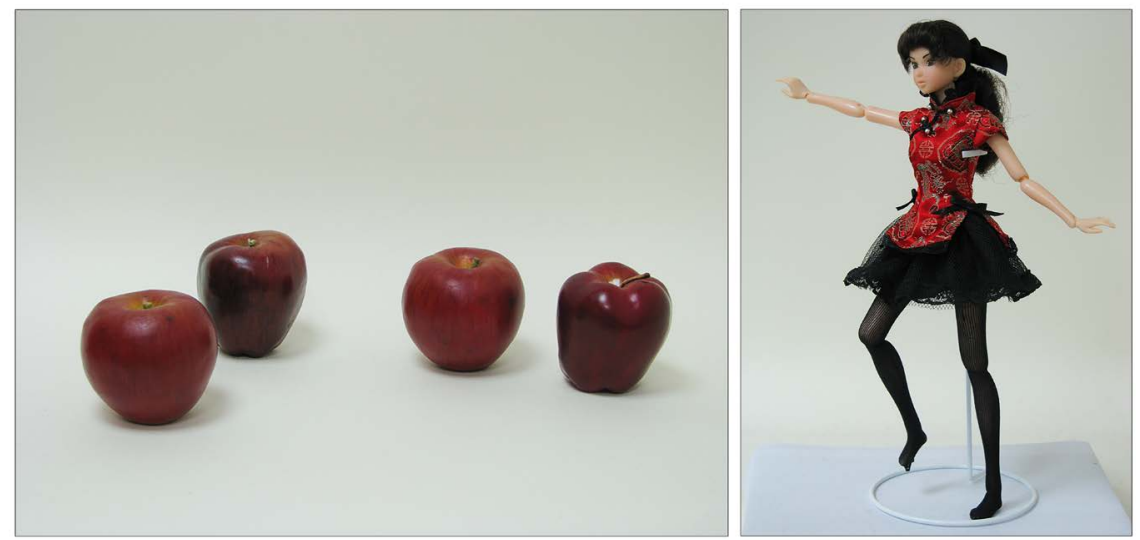

Figure 4. Still-lifes used in Task \#3.

\subsubsection{Drawing Tools and Supplies}

Participants were seated a meter across from the still-life object. The table on which the inanimate object was laid was of the same height as the participant's desk. Participants worked on sheets of white paper $(393 \times 273 \mathrm{~mm})$ with a $2 \mathrm{~B}$ pencil and an eraser.

\subsection{Data Evaluation}

Three expert raters were asked to score the efforts of the participants in each of the three measurements. Expert raters have received professional training in art creation more than 10 years. Their current occupation are art teachers, and have experience of evaluating drawings.

Task \#1: All 3060 lines completed by 51 participants were randomly mixed for scoring. Each line was scored on a 7-point scale (1-weak to 7-strong) in terms of accuracy in position and angle. Raters scored the work by each participant using Photoshop CS6. Before scoring, we overlaid a correct version onto the layer completed by each participant. The frames of both layers were meticulously aligned. Raters could open or close the correct version. We then collated the scores to obtain the mean value of the 82 lines done by each participant to determine absolute positioning ability.

Task \#2: All 153 pictures completed by 51 participants were randomly mixed for scoring. Each picture was scored on a 7-point scale (1-weak to 7-strong) in terms of accuracy. Raters scored all pictures using Photoshop CS6. Before scoring, we overlaid a correct version onto the layer completed by each participant. This version was located by selecting one line that was overlaid exactly. Raters were able to move the correct version as needed. We then collated the scores to obtain the mean value of the three pictures done by each participant to determine relative positioning ability.

Task \#3: The 102 pictures completed by 51 participants were randomly mixed for scoring. Each picture was scored on a 7-point scale (1-weak, 7-strong) in terms of accuracy. This was judged in terms of the relative positions of each object, their forms and shapes, shadows and materials, and realistic representation in its entirety. Emotive inputs and creativity should be excluded from considera- 
tion when making scores. Lastly we obtained the mean values of both picturesfour apples and the action figure-which quantified the realistic drawing ability of each participant.

\section{Results \& Discussion}

\subsection{Inter-Rater Reliability}

All the scoring results were produced by professional raters. We calculated the standard score (z-score) as a way to analyze the aforesaid three abilities. We used Pearson's correlation to evaluate the inter-rater reliability. The inter-rater reliabilities for all three measurements are listed in Tables 1-3. Overall, the ratings given by the three raters are highly correlated.

\subsection{Correlation of Absolute Positioning Ability, Relative Positioning Ability, and Realistic Drawing Ability}

The correlation coefficient of absolute positioning ability and realistic drawing ability is $\mathrm{r}=0.40(\mathrm{n}=51, p=0.004)$, whereas the correlation coefficient of relative positioning ability and realistic drawing ability is $\mathrm{r}=0.37(\mathrm{n}=51, p=$ 0.008). A scatter plot summarizes the results (Figure 5). Both indicate a positive correlation between positioning abilities and realistic drawing ability. Thus our hypothesis that both absolute positioning ability and relative positioning ability are correlated to the degree of accuracy exhibited in a realistic drawing is sup-

Table 1. Inter-rater reliability of Task \#1.

\begin{tabular}{lll}
\hline & & Task \#1 \\
\cline { 3 - 4 } & Rater1 & Rater2 \\
\hline Rater2 & $0.79^{* *}$ & \\
Rater3 & $0.85^{\star *}$ & $0.89^{* *}$ \\
\hline
\end{tabular}

${ }^{* *}$ Correlation is significant at the 0.01 level (2-tailed).

Table 2. Inter-rater reliability of Task \#2.

\begin{tabular}{lll}
\hline & & Task \#2 \\
\cline { 2 - 3 } & Rater1 & Rater2 \\
\hline Rater2 & $0.71^{\star *}$ & \\
Rater3 & $0.89^{\star *}$ & $0.63^{\star *}$ \\
\hline
\end{tabular}

${ }^{* *}$ Correlation is significant at the 0.01 level (2-tailed).

Table 3. Inter-rater reliability of Task \#3.

\begin{tabular}{lll}
\hline & & Task \#3 \\
\cline { 3 - 3 } & Rater1 & Rater2 \\
\hline Rater2 & $0.90^{* *}$ & \\
Rater3 & $0.85^{* *}$ & $0.87^{* *}$ \\
\hline
\end{tabular}

${ }^{* *}$ Correlation is significant at the 0.01 level (2-tailed). 


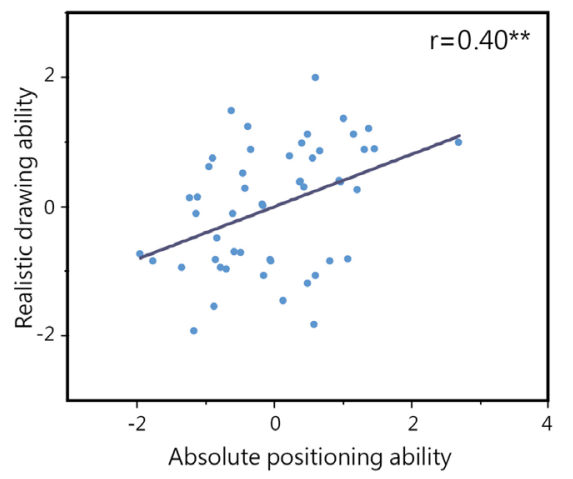

(a)

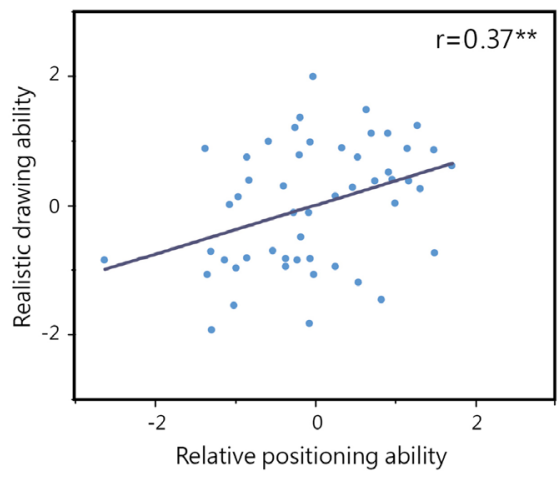

(b)

Figure 5. Scatterplot: (a) absolute positioning ability vs realistic drawing ability; (b) relative positioning ability vs realistic drawing ability.

ported. That is, the better one's absolute positioning ability, the more sophisticated one's production of realistic drawings. The same principle applies to relative positioning ability.

The correlation coefficient of absolute positioning ability and relative positioning ability is $\mathrm{r}=0.16(\mathrm{n}=51, p=0.263)$, which indicates no correlation. A scatter plot summarizes the results (Figure 6). We then used two-Way ANOVA to test the interaction between the two $2 \mathrm{D}$ positioning skills. No significant interactions were observed for absolute positioning and relative positioning ( $\mathrm{F}$ (1, $49)=0.05 ; p=0.83)$. Therefore we conclude that these abilities are independent of each other.

\subsection{Differences between Experts and Novices in Observation Skills}

An Independent-Sample $\mathrm{T}$ Test was conducted to compare realistic drawing performance in experts and novices. There was a significant difference in the scores between the expert group $(M=0.64, \mathrm{SD}=0.71)$ and the novice group ( $M$ $=-0.38, \mathrm{SD}=0.88) ; \mathrm{t}(49)=4.27, p=0.00$.

We conducted an Independent-Sample T Test in experts' and novices' absolute positioning abilities. Results indicate a significant difference in the absolute positioning abilities of the expert group $(\mathrm{M}=0.37, \mathrm{SD}=0.95)$ and their novice counterparts $(\mathrm{M}=-0.22, \mathrm{SD}=0.89) ; \mathrm{t}(49)=2.23, p=0.03$. However, an Independent-Sample $\mathrm{T}$ test showed no significant difference in the relative positioning abilities of experts $(\mathrm{M}=0.25, \mathrm{SD}=0.80)$ and novices $(\mathrm{M}=-0.17, \mathrm{SD}=0.97)$; $\mathrm{t}(49)=1.58, p=0.12$.

The results of Independent-Sample T Test indicate that participants from the expert group outperformed their counterparts in the novice group in realistic drawing and absolute positioning abilities. Yet there was little disparity in terms of relative positioning ability between the two groups. We presume this has something to do with the existence of more referential points in relative positioning. More referential point serves as a clue, thus reducing the difficulty of the task. Even a novice can locate an exact position when there are sufficient referential points. 


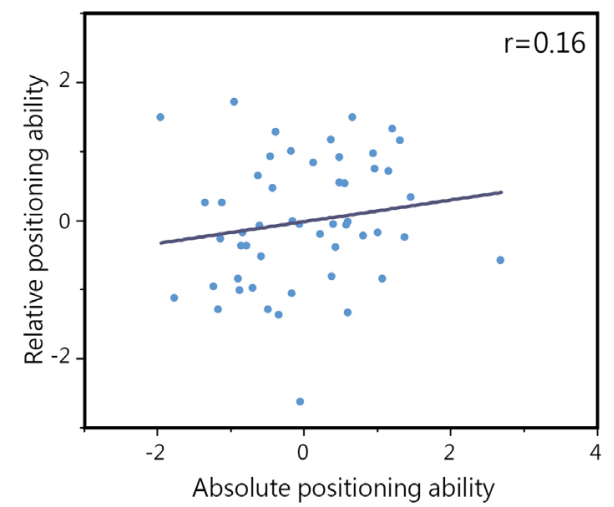

Figure 6. Scatterplot of absolute positioning ability vs relative positioning ability.

In the measurement of absolute positioning ability, participants could only use the frame (indicating the boundary of the canvas) as a referential point. Without the chance to cross-reference, the difficulty of the task increased. This suggests this task is more discriminating. In this task, the expert group outperformed their counterparts.

\section{Results \& Discussion}

Although we depend on visual processing in our everyday lives, the skills required to draw a realistic artwork require much more sophisticated observational strategies than we normally employ. Artists need to closely observe the proportions of the target objects, their relative positions, and even the relationships among the objects and the surrounding space. This study validates the observational strategies used in $2 \mathrm{D}$ positioning processes prior to sketching. An artist uses different referential points as clues within the space so as to locate the exact position for the object yet to be drawn. This study assesses two 2D positioning methods: absolute positioning (which uses the boundary of the canvas as the sole referential point), and relative positioning (which uses the already-sketched lines or objects as a referential system).

Results indicate that these two 2D positioning skills are quite different. They both help to improve the production of a realistic drawing. That is, the better one's absolute positioning ability or relative positioning ability, the more sophisticated one's performance of realistic drawings. This result can be applied in the instruction of realistic drawing skills. When one is in good command of $2 \mathrm{D}$ positioning skills, one may find it easier to locate the correct position of an object and render more satisfactory realistic drawings. However, absolute positioning is a relatively specialized skill, while one does not need a lot of practice to acquire relative positioning ability. With regard to absolute positioning, the differences shown in novices and experts might be due to the reasons stated below. The first possibility is that absolute positioning calls for more talent and on average the experts are more gifted than the novices. The second possibility is that absolute positioning ability requires more intense training to develop, and the experts have received more training and practiced for longer. It is also possible that the 
observed difference is as a result of a combination of contributions from both talent and training (with differing proportions of influence). If "absolute positioning" is generally considered an endowment or gift, then it should be measured by aptitude testing, rather than time spent in training. Contrarily, if the ability of "absolute positioning" can be acquired, then we can predict that performance in this skill is affected by the length of time spent in training, irrespective of one's score in the aptitude test. Given the scope of information garnered for this study, we cannot determine which influence is bigger. This is an area for further research.

\section{Acknowledgements}

This study was supported by grants from Taiwan's Ministry of Science and Technology to IC (MOST 103-2410-H-009-042-). The authors would like to thank all colleagues and students who contributed to this study. We thank Dr. Chia Ning Liao for helping in data analysis. We would like to thank two anonymous (unknown) reviewers and the editor for their comments.

\section{References}

Carson, L., \& Allard, F. (2013). Angle-Drawing Accuracy as an Objective PerformanceBased Measure of Drawing Expertise. Psychology of Aesthetics, Creativity and the Arts, 7, 119-129. https://doi.org/10.1037/a0030587

Cohen, D. J. (2005). Look Little, Look Often: The Influence of Gaze Frequency on Drawing Accuracy. Perception and Psychophysics, 67, 997-1009. https://doi.org/10.3758/BF03193626

Cohen, D. J., \& Bennett, S. (1997). Why Can't Most People Draw What They See. Journal of Experimental Psychology, 23, 609-621. https://doi.org/10.1037/0096-1523.23.3.609

Cohen, D. J., \& Earls, H. (2010). Inverting an Image Does Not Improve Drawing Accuracy. Psychology of Aesthetics, Creativity, and the Arts, 4, 168-172. https://doi.org/10.1037/a0017054

Cohen, D. J., \& Jones, H. E. (2008). How Shape Constancy Relates to Drawing Accuracy. Psychology of Aesthetics, Creativity, and the Arts, 2, 8-19. https://doi.org/10.1037/1931-3896.2.1.8

Day, R. H. (1972). The Basis of Perceptual Constancy and Perceptual Illusion. Investigative Ophthalmology, 11, 525-532.

Kozbelt, A. (2001). Artists as Experts in Visual Cognition. Visual Cognition, 8, 705-723. https://doi.org/10.1080/13506280042000090

Kozbelt, A., \& Seeley, W. P. (2007). Integrating Art Historical, Psychological, and Neuroscientific Explanations of Artists' Advantages in Drawing and Perception. Psychology of Aesthetics, Creativity, and the Arts, 1, 80-90. https://doi.org/10.1037/1931-3896.1.2.80

Kozbelt, A., Seidel, A., El Bassiouny, A., Mark, A., \& Owen, D. R. (2010). Visual Selection Contributes to Artists' Advantages in Realistic Drawing. Psychology of Aesthetics, Creativity, and the Arts, 4, 93-102. https://doi.org/10.1037/a0017657

McManus, I. C., Loo, P. W., Chamberlain, R., Riley, H., \& Brunswick, N. (2011). Does Shape Constancy Relate to Drawing Ability? Two Failures to Replicate. Empirical Studies of the Arts, 29, 191-208. https://doi.org/10.2190/EM.29.2.d

Mitchell, P., Ropar, D., \& Ackroyd, K. (2005). How Perception Impacts on Drawings. 
Journal of Experimental Psychology: Human Perception and Performance, 31, 9961003. https://doi.org/10.1037/0096-1523.31.5.996

Ostrofsky, J., Kozbelt, A., \& Cohen, D. J. (2015). Observational Drawing Biases Are Predicted by Biases in Perception: Empirical Support of the Misperception Hypothesis of Drawing Accuracy with Respect to Two Angle Illusions. The Quarterly Journal of EXperimental Psychology, 68, 1007-1025. https://doi.org/10.1080/17470218.2014.973889

Ostrofsky, J., Kozbelt, A., \& Seidel, A. (2012). Perceptual Constancies and Visual Selection as Predictors of Realistic Drawing Skill. Psychology of Aesthetics, Creativity, and the Arts, 6, 124-136. https://doi.org/10.1037/a0026384

Perdreau, F., \& Cavanagh, P. (2011). Do Artists See Their Retinas? Frontiers in Human Neuroscience, 5, 171. https://doi.org/10.3389/fnhum.2011.00171

Schlewitt-Haynes, L. D., Earthman, M. S., \& Burns, B. (2002). Seeing the World Differently: An Analysis of Descriptions of Visual Experiences Provided by Visual Artists and Nonartists. Creativity Research Journal, 14, 361-372.

https://doi.org/10.1207/S15326934CRJ1434 7

Todorovic, D. (2002). Constancies and Illusions in Visual Perception. Psihologija, 35, 125-207. https://doi.org/10.2298/PSI0203125T

Todorovic, D. (2010). Context Effects in Visual Perception and Their Explanations. Review of Psychology, 17, 17-32.

Submit or recommend next manuscript to SCIRP and we will provide best service for you:

Accepting pre-submission inquiries through Email, Facebook, LinkedIn, Twitter, etc. A wide selection of journals (inclusive of 9 subjects, more than 200 journals) Providing 24-hour high-quality service User-friendly online submission system Fair and swift peer-review system Efficient typesetting and proofreading procedure Display of the result of downloads and visits, as well as the number of cited articles Maximum dissemination of your research work

Submit your manuscript at: http://papersubmission.scirp.org/

Or contact adr@scirp.org 